\title{
YouTube as a Source of Information About Air Pollution
}

\author{
Erhan Kaya ${ }^{1} \cdot$ Musa Şahin ${ }^{2}(\mathbb{0}$
}

Received: 22 November 2021 / Revised: 5 January 2022 / Accepted: 20 January 2022 / Published online: 16 February 2022

(c) The Author(s) under exclusive licence to Institute of Earth Environment, Chinese Academy Sciences 2022

\begin{abstract}
YouTube is an important source of information on air pollution. The information presented on youtube may be of high or low quality. In our study, we aimed to evaluate the YouTube videos for their qualities and compare their view numbers, likes, and comment numbers according to high- and low-quality groups. In our study, $32.2 \%$ of all videos were in the high-quality group, $15.1 \%$ in the intermediate quality group, and $52.7 \%$ in the low-quality group according to Global Quality Scale (GQS). Significant differences were found among the quality groups in video length $(p<0.001)$, dislikes/day $(p=0.043)$, comment/ day $(p=0.005)$, DISCERN (DS) tool scores $(p<0.001)$. The duration and DS tool scores were higher in high-quality videos; dislikes/day and comment/day averages were higher in low-quality videos. On the other hand, there was no significant difference in views, views/day, likes/day, likes ratios among quality groups. On this important matter, low-quality videos are abundant. Considering that there are lots of nonreliable information on YouTube, it is needed that individuals should be referred to reliable videos on air pollution.
\end{abstract}

Keywords YouTube $\cdot$ Air pollution $\cdot$ Information $\cdot$ Quality

\section{Introduction}

At present, air pollution is a big environmental problem that is documented to affect general health, making itself even more serious in developing countries (Dehghan et al. 2018; López-Villarrubia et al. 2010). In 2019, World Health Organization named air pollution and global warming in the top list of the ten causes that threaten human health (World Health Organization 2019). Air pollution has acute and chronic effects on human health ranging from the irritation of the upper respiratory tract, asthma, chronical bronchitis, other pulmonary diseases, and health problems that affect multiple systems and organs (Kampa and Castanas 2008). Air pollution is a serious preoccupation for human health is caused by reasons such as the rapid increase of human population, industrialization, an increase of the demands on

Erhan Kaya

erhan.ky1@gmail.com

Musa Şahin

dr.musasahin@hotmail.com

1 Department of Public Health, Public Health Directorate of Osmaniye, Osmaniye, Turkey

2 Department of Public Health, Public Health Directorate of Adana, Adana, Turkey energy, deforestation, and great accumulation of vehicles in urban areas. In various studies conducted worldwide, air pollution has been associated with high mortality (Rajper et al. 2018).

Even though the perception of people on air pollution has improved over several decades, some gaps in our knowledge of how to mitigate the negative effects of air pollution remain (Larkin and Hystad 2017). In means of communication, the acceleration of air pollution gets widespread coverage in rapidly industrializing cities (Shi et al. 2014). As a means of communication, the internet has become an important means of getting informed as a result of its usage in the communities. At present, YouTube is the most visited website after Google and contains so many videos. YouTube is a popular, free-for-use, widely used video-sharing site that allows users to upload and watch videos (Sahin et al. 2019; Kocyigit et al. 2019; Lewis et al. 2012). Although Youtube is an important source of information for people, video parameters such as view numbers, likes and comments might not result to direct people to qualified and correct videos. So far, no studies have been conducted to evaluate its videos on air pollution. In our study, we aimed to evaluate the videos for their qualities and compare their view numbers, likes, and comment numbers according to high and low-quality groups. 


\section{Material and Methods}

The video-sharing website YouTube (www.youtube.com) was searched from its inception through February 24, 2021 using the keyword "air pollution". The authors utilized keywords such as "air pollution environmental health", "air pollution environmental pollution", and "air pollution public health" to compass environmental and public health videos. A total of 400 videos were selected, with 100 videos from each search term. Selected videos were saved for later evaluation browsing history deleted before searching to avoid being affected by previous searches and the results of the search were listed in order of views count. Duration of the video, the number of views, comments, likes, and dislikes were recorded. The comments per day and views per day of the videos were calculated according to the total number of days the video stayed on YouTube. English language videos were assessed by two public health specialists. Any disagreement between reviewers was resolved by the researchers gathering again and reaching the final decision. Videos that were irrelevant, non-English, duplicate, and longer than one hour were excluded from the study. The remaining 258 videos were analyzed. These videos should contain information about the definition, effects, preventions, causes, measurements, and severity of air pollution. Video sources were categorized into seven headings; Physician/engineer (1), university (2), independent users/blog (3), news agencies (4), health information websites (5), government/organization (6), TV films, documentary (7).

\subsection{Assessment of Quality and Reliability}

*We used the Global Quality Scale (GQS) to assess of quality of the videos. GQS tool designed by Bernard ranges from 1 to 5 points (Bernard et al. 2007). A video's score of 4 or 5 points is considered high quality, 3 points intermediate quality, and 1 or 2 points low quality (Table 1). Some previous studies made with similar methods were observed (Kocyigit et al. 2019; Rittberg et al. 2016).

*We used the modified DISCERN tool (DS) to score the reliability of the videos. DS tool score adapted by Singh ranges from 0 to 5 points. In this tool, each yes answer is given one point and consists of 5 questions in total (Singh et al. 2012; Radonjic et al. 2020) (Table 2).
Table 2 Modified DISCERN reliability tool

1. Are the aims clear and achieved?

2. Are reliable sources of information used?

3. Is the information presented balanced and unbiased?

4. Are additional sources of information listed for patient reference ?

5. Are areas of uncertainty mentioned?

\subsection{Ethics}

Ethics committee approval was not required as public available data were used.

\subsection{Statistical Analysis}

Data were analyzed with SPSS $15 \mathrm{v}$. Chicago, IL. $p$-value $<0.05$ was considered statistically significant. Cohen's kappa coefficient was used for inter-rater reliability. Median (minimum, maximum) indication has been preferred because there are excessive values in some data. The Kolmogorov- Smirnov Test tested for normality, then the Kruskal Wallis test found differences for more than two categorical variables.

\section{Results}

After the inclusion and exclusion criteria were applied, there were 258 videos left for later analysis (Fig. 1). The Kappa scores used to examine inter-rater agreement were 0.81 and 0.84 for the GQStool and DS tool, respectively. The most mentioned topics in the videos were the causes of air pollution $76.4 \%(n=197)$ and the effects of air pollution $75.6 \%$ $(n=195)$ (Table 3).

When the videos were analyzed according to their source, 9.7\% $(n=25)$ of the videos were by physician/engineer, $5.4 \%$ $(n=14)$ by the university, $42.2 \%(n=109)$ by independent users/engineers. blog, $24.1 \%(n=62)$ by new sagencies, $1.2 \%(n=3)$ by health information websites, $\% 2.7(n=7)$ by TV films, documentary and $14.7 \%(n=38)$ shared by government/organization.

$32.2 \%(n=83)$ of all videos were in the high-quality group, $15.1 \%(n=39)$ in the intermediate quality group,

Table 1 Global quality scale

\footnotetext{
1. Poor quality, poor flow, most information missing, not helpful for patients

2. Generally poor, some information is given but of limited use to patients

3. Moderate quality, some important information is adequately discussed

4. Good quality good flow, most relevant information is covered, useful for patients

5. Excellent quality and excellent flow, very useful for patients
} 
Table 3 Video content in air pollution, $n(\%)$

\begin{tabular}{lll}
\hline Contents $^{\mathrm{a}}$ & $n$ & $\%$ \\
\hline Definition & 101 & 39.1 \\
Effect & 195 & 75.6 \\
Prevention & 116 & 45.0 \\
Causes & 197 & 76.4 \\
Measurement & 28 & 10.9 \\
Severity & 95 & 36.9 \\
\hline
\end{tabular}

${ }^{\mathrm{a}}$ There is more than one topic, $n$ : number, \%: percentage and $52.7 \%(n=136)$ in the low-quality group (Table 4$)$. The highest percentage of high-quality video sources were physician/engineer $(64.0 \%)$, government/organization (39.5\%) and university $(35.7 \%)$.

Significant differences were found among the quality groups in video length $(p<0.001)$, dislikes/day $(p=0.043)$, comment/day $(p=0.005)$, DS tool scores $(p<0.001)$. The duration and DS tool scores were higher in high-quality videos; and dislikes/day, comment/day averages were higher in low-quality videos. On the other hand, there was no significant difference in views, views/day, likes/day, likes ratios among quality groups. The like ratio was defined as like/ [like + dislike $]$ (Table 5).

\section{Discussion}

Air pollutants are not always visible and may lead to people is not aware of the air pollution (Sun and Li 2019). In a study conducted in China, it has been that people may develop a high sense of awareness of air pollution through the practices of environmental protection and policies (Dong et al. 2019). In densely populated urban areas, online platforms and digital technology are used as additional resources to facilitate monitoring and detection of air pollution (Hswen et al. 2019; Zhang et al. 2014). The public primarily uses $\mathrm{TV}$, radio, and the internet as a source of information about air pollution has been found out (Liu et al. 2016) in China, one of the countries with the most widespread urban air pollution. In our present world where internet use increases, individuals tend to use online sources about health. YouTube is a significant online platform that is easily accessible and capable of providing information. Today, in YouTube videos, there is no regulation of information. Any person can upload air pollution-related videos accessible to billions of people. Due to that, wrong information spread through this platform might result in worse health consequences (Delli et al. 2016; Szmuda et al. 2020).

In our study, it has been seen that in the videos included, the causes and effects of air pollution were discussed. The
Table 4 Quality categorization of the videos according to video sources, $\mathrm{n}$

\begin{tabular}{lllll}
\hline Video source & Low quality & $\begin{array}{l}\text { Intermediate } \\
\text { quality }\end{array}$ & High quality & Total \\
\hline Physician/engineer & 4 & 5 & 16 & 25 \\
University & 7 & 2 & 5 & 14 \\
Independent users/blog & 55 & 14 & 40 & 109 \\
News agencies & 48 & 9 & 5 & 62 \\
Health information web sites & 1 & 1 & 1 & 3 \\
Government/organization & 15 & 8 & 15 & 38 \\
TV films, documentary & 6 & - & 1 & 7 \\
Total & 136 & 39 & 83 & 258 \\
\hline
\end{tabular}

Table 5 Analysis of video characteristics according to GQS quality, median (minimum-maximum)

\begin{tabular}{lllll}
\hline & Low $(n=136)$ & Intermediate $(n=39)$ & High $(n=83)$ & $p$ value \\
\hline Video length (min) & $4.24(0.50-58.30)$ & $3.30(1.12-48.85)$ & $9.15(0.50-58.20)$ & $<5,300(2434-3,274,764)$ \\
Views & $64,605(2328-6,770,360)$ & $34,458(2389-4,420,640)$ & 0.318 \\
Views/day & $70.14(0.75-5665.36)$ & $24.42(0.69-24.015)$ & $39.59(0.96-2121.46)$ & 0.193 \\
Likes/day & $0.64(0-70.68)$ & $0.12(0-317)$ & $0.29(0-48.28)$ & $0.02(0-2.36)$ \\
Dislikes/day & $0.06(0-2.39)$ & $0.01(0-688)$ & $0.92(0.71-1)$ & $\mathbf{0 . 0 4 3}$ \\
Likes ratio $^{\text {a }}$ & $0.93(0,63-1)$ & $0.92(0.32-0.99)$ & $0.02(0-4.28)$ & 0.874 \\
Comments/day & $0.06(0-13,25)$ & $0,01(0-514)$ & $4(2-5)$ & $\mathbf{0 . 0 0 5}$ \\
DISCERN score & $1(0-4)$ & $3(2-5)$ & & $<\mathbf{0 . 0 0 0 1}$ \\
\hline
\end{tabular}

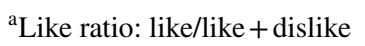


definition of air pollution, its prevention, severity, and measurement were discussed less because the majority of air pollution-related video content on YouTube had been found neither well-organized nor comprehensive on evaluation, even if they did not contain any misleading information. Knowing the sources and their effects on health might increase awareness among people, expand the preventive measures against air pollution and contribute to the reduction of air pollution.

In our study, $32.2 \%$ of the videos were of high quality, $15.1 \%$ were of medium quality and $52.7 \%$ of them were of low quality. In Önder et al.study (Onder and Zengin 2021), while consecutively the high, medium, and low-quality videos were $57.9 \%, 28.9 \%$, and $13.2 \%$, in Koçyiğit et al. (2020) study these rates were $41.4 \%, 21.7 \%$, and $36.9 \%$. In these studies that were conducted on different topics and with similar methods, YouTube could present high-quality information with different rates and additionally, the evaluation of the videos is made based on certain criteria, it is subjective. YouTube contains a significantly high number of videos of low quality depending on sources. At this point, individuals can recognize high-quality videos. The sources of the videos can be an important indicator of quality. In our study, the highest quality video sources consecutively were physician/engineer (64.0\%), government/organization (39.5\%) and university (35.7\%). Examining the other studies, similar to our study, universities, Professional organizations, physician, health information websites, government agencies sources were shown to contain a significantly high number of qualified information (Lewis et al. 2012; Gul and Diri 2019; Kovalski et al. 2019). Individuals should take into consideration the sources of the videos using YouTube, at the same time, they should know that every source can not contain precise and high-quality information. Before all, the users should consider the videos coming from universities, governments, and physicians. Besides, universities, government, and physicians/engineers should upload more videos.

In YouTube, the users can manifest their opinions by clicking 'like' or 'dislike'. In our study, when comparing some parameters according to quality groups, in highquality videos the duration of the video was longer and the DS tool score was higher, in low-quality videos dislikes/ day, comments/day averages were significantly high. In our study, even though viewing numbers did not display much of a difference, view numbers were higher in low-quality videos. In Koçyiğit et al. (Kocyigit et al. 2019) study, while it has been seen that dislike averages were higher in low-quality videos just like in our study, in Şahin et al. (2019) study, in high-quality groups videos were longer. Besides, in these two studies, similarly to our study, viewing numbers, likes, comments did not show great differences according to the quality of the video. The study conducted on the videos about food poisoning, for the quality groups, likes, and viewing numbers were not different $(\mathrm{Li}$ et al. 2019). In the study that oral leukoplakia and video qualities were compared, higher quality videos were liked more. According to the DS tool, high-quality videos had a higher number of likes and viewings (Kovalski et al. 2019). In the study about COVID-19 and rheumatic disease, among the quality groups, in terms of daily viewings, a significant difference was detected no significant difference was detected in daily comments (Kocyigit et al. 2020). In the study about premature ejaculation treatment, videos were qualified as reliable and nonreliable. But, while reliable videos received more likes and fewer dislikes, they received fewer comments (Gul and Diri 2019) In our study as well, it has been detected that low-quality videos received more comments. This situation might be caused by the fact that wrong information awakens a sense of argumentation in individuals. High-quality videos might not manifest significant differences in likes, dislikes, and viewing numbers, low-quality videos might receive more likes and viewings. For this reason, these video parameters should not be used as an indication in YouTube videos and should not be a guide to determinate the right guide for the information. These parameters are dynamic and constantly changing spectator reactions.

This study has some limits. First of all, even though there are some criteria, the evaluation is subjective as there are no confirmed tools yet to evaluate video data. Second of all, YouTube search results are dynamics and the ranking might change adding and deleting videos, and search results might be affected by the ranking standard. Videos were chosen at a specific date for a moment. Besides these, the videos were evaluated in English. For this reason, results might not reflect the whole picture. Because of our search terms, we might have missed some videos. Videos that went beyond the first page were included and different search terms were used, to be able to eliminate the effects of these limitations, in our study. Of the limitations, the dynamic nature of Youtube may have more affected inference. A study with a similar method at another time may give a different perspective.

\section{Conclusion}

As a result, there is a lot of information on air pollution on YouTube. Videos on air pollution provide information to prevent air pollution and mitigate its effects. On this important matter, low-quality videos are abundant. Considering that there are lots of nonreliable information on YouTube, it is needed that individuals should be referred to reliable videos on health information. User choice is the key to accessing qualified information. For video choices, apart from video sources, no parameters should be considered as a quality criterion, universities, government, and physicians/ 
Fig. 1 The process of study

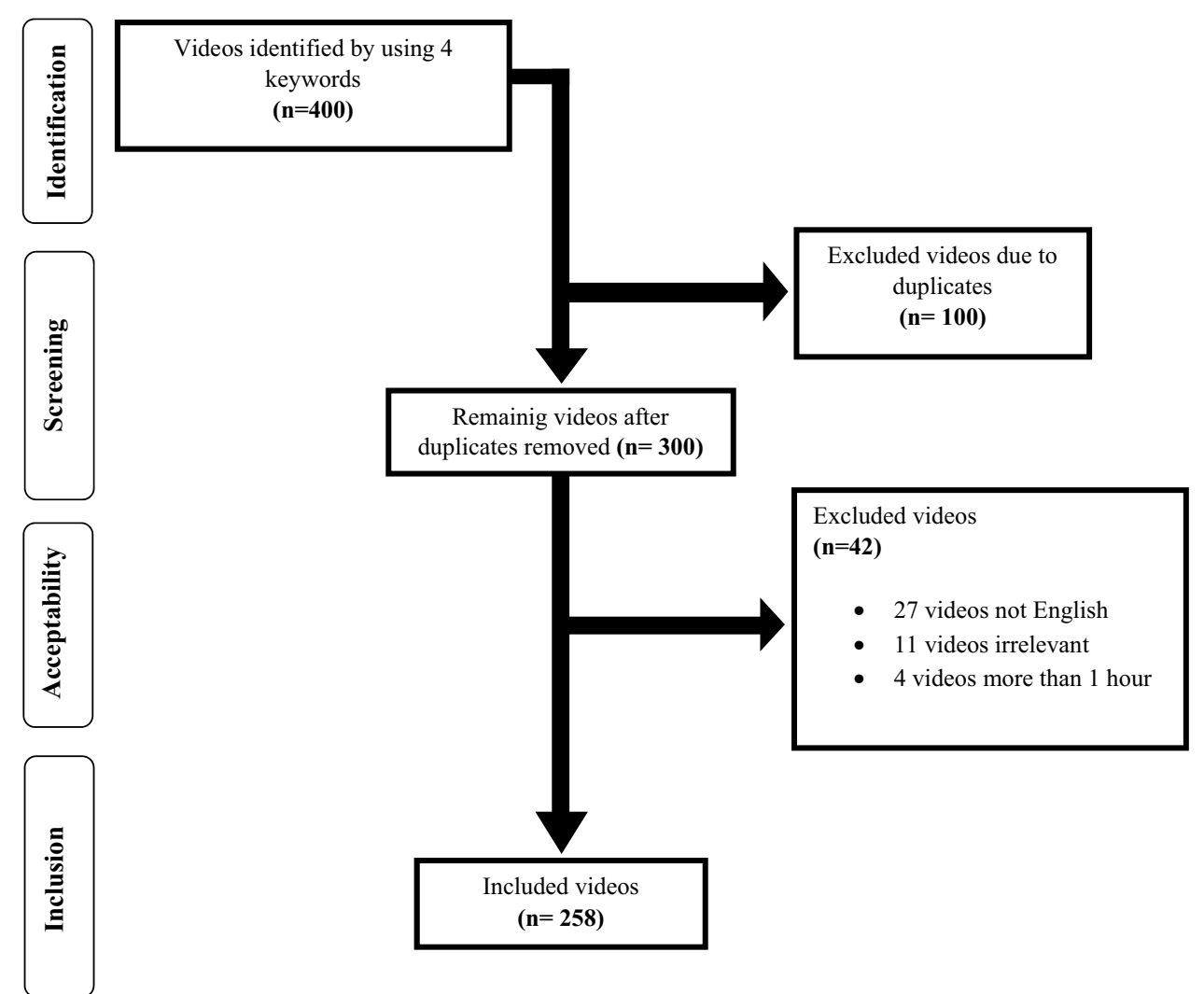

engineers should use YouTube more actively so that users can have more access to precise information.

Author contributions EK and MŞ conceived and supervised the study. EK conducted the analysis. All authors reviewed and edited the manuscript.

Funding None.

Availability of data and material Not applicable.

Code availability Not applicable.

\section{Declarations}

Conflict of interest Not declared.

Ethical approval Not applicable.

Consent to participate Not applicable.

Consent for publication Not applicable.

\section{References}

Bernard A, Langille M, Hughes S, Rose C, Leddin D, Van Zanten SV (2007) A systematic review of patient inflammatory bowel disease information resources on the World Wide Web. Am J
Gastroenterol 102:2070-2077. https://doi.org/10.1111/j.15720241.2007.01325.x

Dehghan A, Khanjani N, Bahrampour A, Goudarzi G, Yunesian M (2018) The relation between air pollution and respiratory deaths in Tehran, Iran-using generalized additive models. BMC Pulm Med 18:1-9. https://doi.org/10.1186/s12890-018-0613-9

Delli K, Livas C, Vissink A, Spijkervet FK (2016) Is YouTube useful as a source of information for Sjögren's syndrome? Oral Dis 22:196-201. https://doi.org/10.1111/odi.12404

Dong D, Xu X, Xu W, Xie J (2019) The Relationship between the actual level of air pollution and residents' concern about air pollution: evidence from Shanghai, China. Int J Environ Res Public Health 16:4784. https://doi.org/10.3390/ijerph16234784

Gul M, Diri MA (2019) YouTube as a source of information about prematüre ejaculation treatment. J Sex Med 16:1734-1740. https:// doi.org/10.1016/j.jsxm.2019.08.008

Hswen Y, Qin Q, Brownstein JS, Hawkins JB (2019) Feasibility of using social media to monitor outdoor air pollution in London, England. Prev Med 121:86-93. https://doi.org/10.1016/j.ypmed. 2019.02.005

Kampa M, Castanas E (2008) Human health effects of air pollution. Environ Pollut 151:362-367. https://doi.org/10.1016/j.envpol. 2007.06.012

Kocyigit BF, Nacitarhan V, Koca TT, Berk E (2019) YouTube as a source of patient information for ankylosing spondylitis exercises. Clin Rheumatol 38:1747-1751. https://doi.org/10.1007/ s10067-018-04413-0

Kocyigit BF, Akaltun MS, Sahin AR (2020) YouTube as a source of information on COVID-19 and rheumatic disease link. Clin Rheumatol 39:2049-2054. https://doi.org/10.1007/ s10067-020-05176-3

Kovalski LNS, Cardoso FB, D'Avila OP, Corrêa APB, Martins MAT, Martins MD, Carrard VC (2019) Is the YouTube ${ }^{\mathrm{TM}}$ a useful source 
of information on oral leukoplakia? Oral Dis 25:1897-1905. https://doi.org/10.1111/odi.13161

Larkin A, Hystad P (2017) Towards personal exposures: how technology is changing air pollution and health research. Curr Environ Health Rep 4:463-471. https://doi.org/10.1007/ s40572-017-0163-y

Lewis SP, Heath NL, Sornberger MJ, Arbuthnott AE (2012) Helpful or harmful? An examination of viewers' responses to nonsuicidal self-injury videos on YouTube. J Adolesc Health 51:380-385. https://doi.org/10.1016/j.jadohealth.2012.01.013

Li M, Yan S, Yang D, Li B, Cui W (2019) YouTube ${ }^{\mathrm{TM}}$ as a source of information on food poisoning. BMC Public Health 19:1-6. https://doi.org/10.1186/s12889-019-7297-9

Liu X, Zhu H, Hu Y, Feng S, Chu Y, Wu Y, Lu Y et al (2016) Public's health risk awareness on urban air pollution in Chinese megacities: The cases of Shanghai, Wuhan and Nanchang. Int J Environ Res Public Health 13(9):845. https://doi.org/10.3390/ijerph1309 0845

López-Villarrubia E, Ballester F, Iñiguez C, Peral N (2010) Air pollution and mortality in the Canary Islands: a time-series analysis. Environ Health 9:1-11. https://doi.org/10.1186/1476-069X-9-8

Onder ME, Zengin O (2021) YouTube as a source of information on gout: a quality analysis. Rheumatol Int. https://doi.org/10.1007/ s00296-021-04813-7

Radonjic A, Hing NNF, Harlock J, Naji F (2020) YouTube as a source of patient information for abdominal aortic aneurysms. J Vasc Surg 71:637-644. https://doi.org/10.1016/j.jvs.2019.08.230

Rajper SA, Ullah S, Li Z (2018) Exposure to air pollution and selfreported effects on Chinese students: a case study of $13 \mathrm{meg}$ acities. PLoS ONE 13:e0194364. https://doi.org/10.1371/journ al.pone.0194364
Rittberg R, Dissanayake T, Katz SJ (2016) A qualitative analysis of methotrexate self injection education videos on YouTube. Clin Rheumatol 35:1329-1333. https://doi.org/10.1007/ s10067-015-2910-5

Sahin AN, Sahin AS, Schwenter F, Sebajang H (2019) YouTube videos as a source of information on colorectal cancer: what do our patients learn? J CancerEduc 34:1160-1166. https://doi.org/10. 1007/s13187-018-1422-9

Shi H, Wang Y, Huisingh D, Wang J (2014) On moving towards an ecologically sound society: with special focus on preventing future smog crises in China and globally. J Clean Prod 64:9-12. https:// doi.org/10.1016/j.jclepro.2013.07.024

Singh AG, Singh S, Singh PP (2012) YouTube for information on rheumatoid arthritis—a wakeup call? J Rheumatol 39:899-903. https:// doi.org/10.3899/jrheum.111114

Sun Z, Li J (2019) Citizens' satisfaction with air quality and key factors in China-using the anchoring vignettes method. Sustainability 11:2206. https://doi.org/10.3390/su11082206

Szmuda T, Syed MT, Singh A, Ali S, Özdemir C, Słoniewski P (2020) YouTube as a source of patient information for Coronavirus Disease (COVID-19): a content-quality and audience engagement analysis. Rev Med Virol 30(5):e2132. https://doi.org/10.1002/ rmv. 2132

World HealthOrganization (2019). Ten threats to global health in 2019. https://www.who.int/news-room/spotlight/ten-threa ts-to-global-health-in-2019

Zhang MW, Ho CS, Fang P, Lu Y, Ho RC (2014) Usage of social media and smartphone application in assessment of physical and psychological well-being of individuals in times of a major air pollution crisis. JMIR Mhealth Uhealth 2(1):e16. https://doi.org/ 10.2196/mhealth.2827 